\title{
Volatile Compounds of Baccharis punctulata, Baccharis dracunculifolia and Eupatorium laevigatum obtained using Solid Phase Microextraction and Hydrodistillation
}

\author{
Patrícia Schossler, ${ }^{a}$ Guilherme Leal Schneider, ${ }^{a}$ Diego Wunsch, ${ }^{a}$ Geraldo Luiz Gonçalves Soares ${ }^{b}$ \\ and Cláudia Alcaraz Zini $*, a$ \\ ${ }^{a}$ Instituto de Química and ${ }^{b}$ Instituto de Biociências, \\ Universidade Federal do Rio Grande do Sul, Av. Bento Gonçalves 9500, 91501970 Porto Alegre-RS, Brazil
}

\begin{abstract}
Neste trabalho foram comparados perfis cromatográficos dos óleos provenientes de hidrodestilação de folhas de Baccharis punctulata, Baccharis dracunculifolia e Eupatorium laevigatum e os perfis cromatográficos obtidos utilizando-se HS-SPME. Várias plantas nativas do Brasil ainda não foram estudadas quanto à sua composição química. Técnicas convencionais de extração como a hidrodestilção, podem resultar em alterações da composição química original destes óleos. O uso da HS-SPME provê uma alternativa mais branda de extração, prevenindo transformações químicas e resultando em informações complementares sobre a composição de voláteis das plantas. Pela primeira vez, cumarina e cumaran foram identificados no "headspace" de folhas de E. laevigatum, após dano mecânico, por HS-SPME. As semelhanças e diferenças na composição dos voláteis, verificadas através de ambas as técnicas de extração, são discutidas, mostrando a complementaridade destas técnicas, as possíveis implicações destes resultados no que diz respeito a compostos infoquímicos e as possíveis transformações químicas durante o processo de hidrodestilação.
\end{abstract}

In this work the qualitative chromatographic profiles of the volatile oil obtained with fresh chopped leaves of Baccharis punctulata, Baccharis dracunculifolia and Eupatorium laevigatum, using HS-SPME were compared with their hydrodistilled oils. Several Brazilian native plant species have not yet been studied regarding their volatile compounds composition. Conventional techniques employed for the investigation of volatile compounds, such as hydrodistillation, may impart chemical changes to the original oil composition. The use of HS-SPME provides alternative milder extraction conditions, preventing chemical transformations and supplying complementary information about volatiles composition. Coumarin and coumaran were detected by the first time among volatile components of E. laevigatum leaves after mechanical damage, only when using HS-SPME. Differences and similarities perceived between volatile compounds profiles using both extraction techniques are discussed, showing that they are complementary and may bring insight about fresh leaf volatiles playing infochemical roles and about chemical transformations caused by hydrodistillation.

Keywords: Baccharis punctulata, Baccharis dracunculifolia, Eupatorium laevigatum, infochemicals, solid phase micro extraction

\section{Introduction}

Plant biogenic volatile organic compounds (BVOC) play several roles related to intra and inter species interactions, and are also important in various branches of industry, such as flavour and fragrances, pesticides, and perfumery industry. ${ }^{1,2}$ Several factors may influence plant volatiles composition, such as freshness, grinding and

\footnotetext{
*e-mail: cazini @iq.ufrgs.br
}

drying processes, environmental conditions, extraction techniques etc. ${ }^{1,3}$ Hydrodistillation (HD) as well as steam distillation (SD) are conventional techniques of isolation of volatile compounds, where temperature and $\mathrm{pH}$ may promote artifact formation, being also time consuming. On the other side, headspace solid phase micro extraction (HS-SPME) provides solventless extractions under mild temperatures and shorter extraction times. ${ }^{1,4,5}$

Several recent research works take advantage of the mild temperatures of HS-SPME to obtain information on 
BVOC of fresh plants ex situ, in order to achieve different purposes. Some examples are: comparison of BVOC released by different parts of various plants ${ }^{6,7}$ analysis of volatile compounds of Eruca sativa, ${ }^{8}$ Hypericum triquetrifolium, ${ }^{9}$ and Myrtus communis ${ }^{10}$ responsible for different aroma notes, and analysis of volatiles of freshcut pineaple during storage or UV-induced stress. ${ }^{11}$ Some reports also compare information gathered with HS-SPME with those obtained using other techniques, such as $\mathrm{HD}^{12,13}$ or $\mathrm{SD}^{14-16}$ or several other extraction techniques. ${ }^{3}$

Former work of this group has already shown the potential of HS-SPME technique to extract compounds that were not found in the hydrodistilled oil of exotic plants (Eucalyptus) and that may play a role as infochemicals. ${ }^{4,5}$

Three Brazilian native species Baccharis punctulata, Baccharis dracunculifolia, and Eupatorium laevigatum (Asteraceae) are widely distributed in the South part of Brazil and were the object of this study. These species represent a social-economic potential because of their therapeutic use related to several diseases. The scientific literature presents some information about the volatile oils of B. dracunculifolia and E. laevigatum, although there is no report about $B$. punctulata essential oil. ${ }^{17-19}$ However, neither one of these species has had their volatile compounds investigated by HS-SPME. The aim of this work is to compare the volatile oil composition of the three just mentioned native species of Rio Grande do Sul state using two different techniques, the conventional hydrodistillation and HS-SPME. Differences and similarities among results obtained with both techniques are discussed.

\section{Experimental}

\section{Plant material}

Adult fresh leaves of B. punctulata were collected in January $26^{\text {th }}, 2005$ and in September, $21^{\text {th }}, 2005$ in the $\mathrm{km}$ 307.5 of the BR 116, nearby Guaiba city in the state of Rio Grande do Sul, Brazil. In both samplings leaves of shrubs were sampled in the right and left side of the BR 116. Adult leaves of three shrubs of $B$. dracunculifolia were collected in the same days in the fields of São Maximiamo Farm (km 308 of BR 116 nearby Guaiba city in state of Rio Grande do Sul). Sampling of E. laevigatum young plants was performed in October $19^{\text {th }}, 2005$ in the same São Maximiamo Farm and its surroundings. Topographic coordinates of São Maximiamo Farm are 30 10' $47^{\prime \prime}$ S and $51^{\circ} 23$ ' $33^{\prime \prime} \mathrm{W}$. Leaves of ten shrubs were sampled in the farm and on the edge of BR 116 road. E. laevigatum adult leaves were sampled in September $7^{\text {th }}, 2004$ by the sides of BR 386 road, approximately $12 \mathrm{~km}$ before Soledade city.
All the samples were identified by Prof. Dr. Nelson Ivo Matzenbacher and a voucher specimen of B. punctulata (ICN 157537), B. dracunculifolia (ICN 143383) and E. laevigatum (ICN 029328) has been deposited at the herbarium of Universidade Federal do Rio Grande do Sul, Brazil.

\section{Hydrodistillation}

Fresh leaves of B. punctulata, B. dracunculifolia and E. laevigatum were subjected to hydrodistillation in a modified Clevenger apparatus for 5 hours. A cooling system using ethylene glycol mixed to water kept condenser temperature between $-2{ }^{\circ} \mathrm{C}$ and $4{ }^{\circ} \mathrm{C}$. Anhydrous sodium sulphate, previously heated to $400{ }^{\circ} \mathrm{C}$, was employed to eliminate essential oil humidity.

\section{$H S-S P M E$}

HS-SPME was performed using fibers coated with $7 \mu \mathrm{m}$ poly (dimethylsiloxane) (PDMS), $0.5 \mathrm{~g}$ of fresh chopped leaves and $4 \mathrm{~mL}$ of phosphate buffer $(\mathrm{pH} 7$ ) inside $10 \mathrm{~mL}$ clear flask vials. Samples were kept at $30{ }^{\circ} \mathrm{C}$ in a temperature-controlled block for a minimum of $24 \mathrm{~h}$, and headspace extractions were performed during $30 \mathrm{~min}$. Fresh leaves used for HS-SPME were from the same batch of leaves used for hydrodistillation. More details on HS-SPME method development are reported in a former research work. ${ }^{4,5}$ Fibers were supplied by Supelco (Oakville, ON, Canada), and were conditioned according to supplier's instructions before use. For each plant species, at least 5 replicates of extraction were performed. For E. laevigatum, chopped leaves headspace from adult and young plants were extracted separately.

\section{Chromatographic analysis}

Chromatographic analyses were performed with a Shimadzu gas chromatograph G17A coupled to a mass spectrometer detector QP 5050A. Two capillary columns were used under the following conditions: (i) OV-5 (Ohio Valley, Marietta, USA, dimensions $30 \mathrm{~m} \times 0.25 \mathrm{~mm} \times$ $0.25 \mu \mathrm{m}$ ); oven temperature programme starting from $60^{\circ} \mathrm{C}$ raising at $3{ }^{\circ} \mathrm{C} \mathrm{min}^{-1}$ to $250{ }^{\circ} \mathrm{C}$; injector and detector were kept at $250^{\circ} \mathrm{C}$; helium flow at $1 \mathrm{~mL} \mathrm{~min}^{-1}$. (ii) Supelcowax 10 (Supelco, Bellefonte, USA, dimensions $30 \mathrm{~m} \times 0.25 \mathrm{~mm} \times$ $0.25 \mu \mathrm{m}$ ); oven temperature programme starting from $40^{\circ} \mathrm{C}$ raising at $3{ }^{\circ} \mathrm{C} \mathrm{min}^{-1}$ to $220{ }^{\circ} \mathrm{C}$; injector and detector were kept at $220^{\circ} \mathrm{C}$; helium flow at $1 \mathrm{~mL} \mathrm{~min}^{-1}$. Injection of a $1 \%$ hexanic solutions of essential oils were made in the split mode (1:10), while HS-SPME analyses were made in the splitless mode and only in the OV5 column. 
The oils were also analyzed in a Shimadzu GC-FID 17A, under similar chromatographic conditions on both columns. Linear temperature programmed retention indices (LTPRI) were calculated using the retention data of a $1 \%$ hexanic solution of linear alkanes (C9 to $\mathrm{C} 24$ ), along with retention data of the compounds of the three essential oils. Identification of volatile components was done comparing injections of pure compounds with unknown ones, keeping the same chromatographic conditions. When pure compounds were not available, comparison with retention data and LTPRI reported in the literature was used for tentative identification of the compounds. Comparison of mass spectra of the $6^{\text {th }}$ edition of the Wiley library and the unknown compounds spectra was also employed for tentative identification. ${ }^{20}$

\section{Results and Discussion}

Differences found in volatile compounds of plants isolated with HS-SPME and conventional methods such as HD and SD are reported in the literature. Sometimes HS-SPME provides a larger scope of compounds, ${ }^{4,21}$ in other cases, HS-SPME detects a lesser amount of compounds ${ }^{22}$ and in some other cases differences found are only quantitative. ${ }^{13}$ Quantitative differences are normally expected when dealing with techniques that are based in different principles of extraction, such as HD (exhaustive) and SPME (equilibrium). However, area percent of volatile components are normally reported to provide the order of magnitude of each compound in the whole mixture, as presented in the Table 1. Besides that, the various fiber coatings present different chemical affinity for the analytes and several extraction parameters (temperature, time, etc) may influence extraction results. Also, distinct plant tissues present different matrix effects on the volatile compounds. ${ }^{1,13,22}$ For all these reasons, HSSPME sampling conditions should be carefully planned in order to obtain meaningful data. The Table 1 shows a total of 122 compounds detected in the three species under study using hydrodistillation and HS-SPME as extraction techniques. Compounds detected by both techniques are 60 for B. punctulata, 42 for B. dracunculifolia and 33 for E. laevigatum. Besides those compounds, 15 more components were found only by HS-SPME in the headspace of E. laevigatum and of B. dracunculifolia.

Other twenty compounds were detected only in the hydrodistilled oil of the species under investigation. In B. punctulata essential oil the main compounds are a non identified sesquiterpene followed by bicyclogermacrene, a sesquiterpene, cis-cadin-4-en-ol, and (Z)-ocimene. The major components of $B$. dracunculifolia and E. laevigatum are nerolidol, an important component for the perfumery industry, ${ }^{17}$ and the oxygenated sesquiterpene laevigatin, respectively. ${ }^{19}$ Figure 1 presents the chromatographic profile of the hydrodistilled oils of $B$. punctulata, $B$. dracunculifolia, and E. laevigatum and also point to some small peaks that were detected only by HD, whose names are written in underlined font.

As the contribution of these peaks are minor and can hardly be seen in Figure 1, they will be highlighted in the following Figures. As it is possible to see from Table 1, the majority of the components of these three species were detected in the headspace of chopped leaves and also in the essential oil. Although differences found between results of both techniques were minor, they may convey meaningful information. It is well known that very small amounts of volatile compounds can act as important signals in the recognition of food source by insects. Some interactions between plants and phytophagous insects involve specific volatile chemical cues in the range of parts per million or even parts per billion of plant weight. ${ }^{23,24} \mathrm{In}$ most insect species food location is heavily dependent on olfactory cues. Some investigations have demonstrated highly specific plant odor neurons responding selectively to single compound at very low concentrations. ${ }^{25-31}$ As an example to this the Melanophila acuminate antennae can detect guaiacol derivatives at concentrations as low as a few parts per billion. ${ }^{24,32}$ Buttery and Ling also detected several hydrocarbons as the major components of a low concentration mixture ( $c a .10$ parts per billion) in corn roots, which acted as attractants of insects, such as corn root worm (Diabrotica spp.). ${ }^{23}$

Figure 2A presents a clearer picture of some compounds detected only by HD in $B$. dracunculifolia in the region where monoterpenes elute. Chromatographic peaks representing $\alpha$-terpinene, $\gamma$-terpinene, terpinolene, and terpinen-4-ol were found only in the hydrodistilled oil and were not detected in the headspace of chopped leaves of the same plant (Figure 2B). A similar pattern is observed for $B$. punctulata, where $\alpha$-terpinene and terpinen-4-ol were also detected only in the essential oil (Table 1). Several researchers have already reported the tendency of sabinene and $\alpha$-thujene to undergo acid catalysed hydration, resulting in $\alpha$-terpinene, terpinolene, $\gamma$-terpinene and terpinen-4-ol. ${ }^{33,34}$ As hydrodistillation is carried out at higher temperatures than SPME and may provide lower $\mathrm{pHs}$ in aqueous medium, the presence of those monoterpenes may be regarded as artifacts of hydrodistillation.

Figure 3 shows some other $B$. dracunculifolia and $B$. punctulata volatile compounds that were detected by only one of the extraction techniques employed and not by the other. 
Table 1. Compounds detected in the headspace of chopped leaves and in the hydrodistilled oil of Baccharis punctulata, Baccharis dracunculifolia, and Eupatorium leavigatum

\begin{tabular}{|c|c|c|c|c|c|c|c|c|c|c|}
\hline \multirow[b]{2}{*}{ No. } & \multirow[b]{2}{*}{ Compound } & \multirow{2}{*}{$\begin{array}{l}\text { LTPRI } \\
\text { OV5 }\end{array}$} & \multirow{2}{*}{$\begin{array}{l}\text { LTPRI } \\
\text { Lit. }^{20}\end{array}$} & \multirow[b]{2}{*}{ LTPRI Wax } & \multicolumn{2}{|c|}{ B. punctulata } & \multicolumn{2}{|c|}{ B. dracunculifolia } & \multicolumn{2}{|c|}{ E. laevigatum } \\
\hline & & & & & $\begin{array}{c}\text { HD } \\
\% \text { area }\end{array}$ & $\begin{array}{l}\text { SPME } \\
\text { \%area }\end{array}$ & $\begin{array}{c}\text { HD } \\
\% \text { area }\end{array}$ & $\begin{array}{l}\text { SPME } \\
\% \text { area }\end{array}$ & $\begin{array}{c}\text { HD } \\
\text { \%area }\end{array}$ & $\begin{array}{l}\text { SPME } \\
\text { \%area }\end{array}$ \\
\hline \multicolumn{2}{|c|}{ monoterpene hydrocarbons } & & & & 20.13 & 16.02 & 31.09 & 46.17 & 1.72 & 0.36 \\
\hline 1 & $\alpha$-thujene & 927 & 930 & 1023 & $0.26^{\mathrm{b}}$ & $0.32^{\mathrm{b}}$ & $0.08^{\mathrm{b}}$ & $0.09^{\mathrm{b}}$ & $0.41^{\mathrm{b}}$ & $0.07^{\mathrm{b}}$ \\
\hline 2 & $\alpha$-pinene & 934 & 939 & 1029 & $0.31^{\mathrm{a} b}$ & $0.39^{\mathrm{a}, \mathrm{b}}$ & $8.00^{\mathrm{b}}$ & $8.70^{b}$ & $0.28^{\mathrm{b}}$ & $0.07^{\mathrm{b}}$ \\
\hline 3 & camphene & 949 & 954 & 1067 & & & $0.06^{\mathrm{b}}$ & $0.11^{\mathrm{b}}$ & & \\
\hline 4 & sabinene & 973 & 975 & 1120 & $0.59^{b}$ & $0.94^{\mathrm{b}}$ & $0.44^{\mathrm{b}}$ & $1.38^{b}$ & $0.09^{\mathrm{b}}$ & $\operatorname{tr}^{\mathrm{b}}$ \\
\hline 5 & $\beta$-pinene & 978 & 979 & 1110 & $0.32^{a b}$ & $0.50^{\mathrm{a} b}$ & $12.17^{b}$ & $14.95^{\mathrm{b}}$ & $0.12^{\mathrm{b}}$ & $\operatorname{tr}^{\mathrm{b}}$ \\
\hline 6 & myrcene & 990 & 991 & 1170 & $0.41^{a b}$ & $0.19^{\mathrm{a} b}$ & $1.99^{\mathrm{b}}$ & $4.73^{b}$ & $0.11^{\mathrm{b}}$ & $\operatorname{tr}^{\mathrm{b}}$ \\
\hline 7 & monoterpene & - & - & - & & & $\operatorname{tr}^{\mathrm{c}}$ & $0.15^{\mathrm{c}}$ & & \\
\hline 8 & $\alpha$-phellandrene & 1004 & 1003 & 1167 & $0.14^{\mathrm{a} b}$ & $\operatorname{tr}^{\mathrm{a}}$ & & & & \\
\hline 9 & $\alpha$-terpinene & 1017 & 1017 & 1183 & $\underline{\mathbf{0 . 0 6}}^{\mathrm{a}}$ & & $\underline{\mathbf{0 . 1 0}}^{\mathrm{a} b}$ & & & \\
\hline 10 & limonene & 1028 & 1029 & 1203 & $6.00^{\mathrm{a} b}$ & $4.21^{\mathrm{a} b}$ & $7.65^{\mathrm{b}}$ & $14.66^{\mathrm{b}}$ & $0.23^{\mathrm{b}}$ & $\operatorname{tr}^{\mathrm{b}}$ \\
\hline 11 & 1,8-cineole & 1028 & 1031 & - & & & $0.05^{\mathrm{b}}$ & $0.90^{\mathrm{b}}$ & & \\
\hline 12 & $(Z)$-ocimene & 1035 & 1037 & 1242 & $6.33^{\mathrm{a}, \mathrm{b}}$ & $7.25^{\mathrm{a} b}$ & $0.40^{\mathrm{b}}$ & $0.50^{\mathrm{b}}$ & & \\
\hline 13 & $(E)$-ocimene & 1046 & 1050 & 1259 & $4.96^{\mathrm{a}, \mathrm{b}}$ & $2.09^{a b}$ & & & $0.39^{\mathrm{b}}$ & $0.11^{\mathrm{b}}$ \\
\hline 14 & $\gamma$-terpinene & 1058 & 1060 & 1249 & $0.09^{\mathrm{a}, \mathrm{b}}$ & $\operatorname{tr}^{\mathrm{a}, \mathrm{b}}$ & $\underline{0.15}^{\mathrm{b}}$ & & $0.05^{\mathrm{b}}$ & $\operatorname{tr}^{\mathrm{b}}$ \\
\hline 15 & terpinolene & 1088 & 1089 & 1288 & $\operatorname{tr}^{\mathrm{a}, \mathrm{b}}$ & $\operatorname{tr}^{\mathrm{a}, \mathrm{b}}$ & $\underline{0.11}^{\mathrm{b}}$ & & $0.05^{\mathrm{b}}$ & $\operatorname{tr}^{\mathrm{b}}$ \\
\hline 16 & linalool & 1097 & 1091 & 1563 & $0.25^{\mathrm{b}}$ & $\operatorname{tr}^{\mathrm{b}}$ & $0.10^{\mathrm{b}}$ & $0.09^{\mathrm{b}}$ & & \\
\hline 17 & monoterpene & 1114 & - & 1312 & $\underline{\operatorname{tr}}^{\mathrm{c}}$ & & & & & \\
\hline 18 & allo ocimene & 1126 & 1132 & - & $0.59^{b}$ & $0.07^{\mathrm{b}}$ & & & & \\
\hline \multicolumn{2}{|c|}{ oxigenated monoterpenes } & & & & 0.61 & 0.05 & 0.72 & 0.26 & & 0.06 \\
\hline 19 & terpin-4-ol & 1178 & 1177 & 1605 & $\underline{\mathbf{0 . 1 4}}^{\mathrm{a} . \mathrm{b}}$ & & $\underline{0.24}^{\mathrm{b}}$ & & & \\
\hline 20 & $\alpha$-terpineol & 1189 & 1189 & - & $0.17^{\mathrm{a} . \mathrm{b}}$ & $\operatorname{tr}^{\mathrm{a} \cdot \mathrm{b}}$ & $0.38^{\mathrm{b}}$ & $0.17^{\mathrm{b}}$ & & \\
\hline 21 & coumaran & - & - & - & & & & & & $\underline{\mathbf{t r}}^{\mathrm{c}}$ \\
\hline 22 & hexenyl ester & - & - & - & & & & & & $\underline{\operatorname{tr}}^{\mathrm{c}}$ \\
\hline 23 & hexenyl ester & - & - & - & & & & & & $\underline{\mathbf{t r}}^{\mathrm{c}}$ \\
\hline 24 & hexenyl ester & - & - & - & & & & & & $\underline{\operatorname{tr}}^{\mathrm{c}}$ \\
\hline 25 & trans-geraniol & 1251 & 1253 & - & $\underline{\mathbf{t r}}^{\mathrm{b}}$ & & & & & \\
\hline 26 & $\begin{array}{l}n q-69(100), 95(85), 55(65), \\
110(45), 152(33)\end{array}$ & 1259 & - & 1315 & & & $0.08^{\mathrm{b}}$ & $0.14^{\mathrm{b}}$ & & \\
\hline \multicolumn{2}{|c|}{ sesquiterpene hydrocarbons } & & & 19.38 & 62.67 & 22.09 & & 25.11 & 82.90 & \\
\hline 27 & $\begin{array}{l}\mathrm{nq}-150(100), 135(93), 107(32) \\
77(26), 151(10)\end{array}$ & 1310 & - & 1412 & $\underline{0.10}$ & & & & & \\
\hline 28 & sesquiterpene & - & - & - & & & & & & $\underline{\operatorname{tr}}^{c}$ \\
\hline 29 & $\delta$-elemene & 1338 & 1338 & 1483 & $0.96^{b}$ & $0.68^{\mathrm{b}}$ & $0.09^{b}$ & $0.15^{b}$ & $0.11^{\mathrm{b}}$ & $0.23^{\mathrm{b}}$ \\
\hline 30 & sesquiterpene & - & - & - & & & & & & $\underline{\operatorname{tr}}^{\mathrm{c}}$ \\
\hline 31 & sesquiterpene & - & - & - & & & & & & $\underline{\operatorname{tr}}^{\mathrm{c}}$ \\
\hline 32 & $\alpha$-ylangene & 1374 & 1375 & - & & & & $\underline{0.20}^{\mathrm{c}}$ & & \\
\hline 33 & $\beta$-bourbonene & 1387 & 1388 & 1520 & & & $0.10^{\mathrm{b}}$ & $0.23^{b}$ & & $\underline{\operatorname{tr}}^{\mathrm{c}}$ \\
\hline
\end{tabular}


Table 1. Continuation

\begin{tabular}{|c|c|c|c|c|c|c|c|c|c|c|}
\hline \multirow[b]{2}{*}{ No. } & \multirow[b]{2}{*}{ Compound } & \multirow[b]{2}{*}{$\begin{array}{l}\text { LTPRI } \\
\text { OV5 }\end{array}$} & \multirow[b]{2}{*}{$\begin{array}{l}\text { LTPRI } \\
\text { Lit. }^{20}\end{array}$} & \multirow[b]{2}{*}{ LTPRI Wax } & \multicolumn{2}{|c|}{ B. punctulata } & \multicolumn{2}{|c|}{ B. dracunculifolia } & \multicolumn{2}{|c|}{ E. laevigatum } \\
\hline & & & & & $\begin{array}{c}\text { HD } \\
\% \text { area }\end{array}$ & $\begin{array}{l}\text { SPME } \\
\text { \%area }\end{array}$ & $\begin{array}{c}\text { HD } \\
\% \text { area }\end{array}$ & $\begin{array}{l}\text { SPME } \\
\% \text { area }\end{array}$ & $\begin{array}{c}\text { HD } \\
\% \text { area }\end{array}$ & $\begin{array}{l}\text { SPME } \\
\% \text { area }\end{array}$ \\
\hline 34 & $\beta$-elemene & 1392 & 1391 & 1596 & $0.39^{\mathrm{b}}$ & $1.13^{\mathrm{b}}$ & $0.41^{\mathrm{b}}$ & $0.35^{\mathrm{b}}$ & $0.23^{\mathrm{b}}$ & $0.21^{\mathrm{b}}$ \\
\hline 35 & $\alpha$-gurjunene & 1411 & 1410 & 1662 & $0.05^{\mathrm{b}}$ & $0.51^{\mathrm{b}}$ & & & & \\
\hline 36 & methyl eugenol & 1413 & 1404 & 2023 & & & $0.20^{\mathrm{b}}$ & $0.11^{\mathrm{b}}$ & & \\
\hline 37 & sesquiterpene & 1415 & - & - & & & & 0.12 & & \\
\hline 38 & (E)-caryophyllene & 1421 & 1419 & 1675 & $0.63^{\mathrm{b}}$ & $6.80^{\mathrm{b}}$ & $2.79^{b}$ & $5.68^{\mathrm{b}}$ & $1.62^{\mathrm{b}}$ & $1.28^{\mathrm{b}}$ \\
\hline 39 & coumarin & - & $1224^{\mathrm{d}}$ & - & & & & & & ${\underline{0.12^{\mathrm{a}}}}$ \\
\hline 40 & $\beta$-copaene & 1430 & 1432 & 1673 & $0.06^{\mathrm{b}}$ & $0.27^{\mathrm{b}}$ & & & & \\
\hline 41 & sesquiterpene & - & - & - & & & & & & $\underline{0.30}^{c}$ \\
\hline 42 & sesquiterpene & 1436 & - & 1697 & $\underline{0.05}^{\mathrm{c}}$ & & & & & \\
\hline 43 & aromadendrene & 1441 & 1441 & 1609 & $\underline{\mathbf{0 . 1 3}}^{\mathrm{ab}}$ & & $0.47^{\mathrm{b}}$ & $0.36^{\mathrm{b}}$ & $\underline{0.08}^{\mathbf{b}}$ & \\
\hline 44 & sesquiterpene & 1442 & - & 1648 & $0.09^{\mathrm{c}}$ & $0.40^{c}$ & & & & \\
\hline 45 & sesquiterpene & 1446 & - & 1689 & & & $\underline{0.20}^{\mathrm{c}}$ & & & \\
\hline 46 & sesquiterpene & 1447 & - & 1645 & & & & & $0.09^{c}$ & $0.23^{\mathrm{c}}$ \\
\hline 47 & sesquiterpene & 1452 & - & - & & & $0.28^{\mathrm{c}}$ & $0.33^{\mathrm{c}}$ & & \\
\hline 48 & $\alpha$-humulene & 1456 & 1455 & 1675 & $0.16^{\mathrm{b}}$ & $0.20^{\mathrm{b}}$ & $0.72^{\mathrm{b}}$ & $0.84^{\mathrm{b}}$ & & \\
\hline 49 & $(E)-\beta$-farnesene & 1457 & 1457 & 1676 & $0.27^{b}$ & $3.89^{\mathrm{b}}$ & & & $0.60^{\mathrm{b}}$ & $1.33^{\mathrm{b}}$ \\
\hline 50 & sesquiterpene & 1460 & - & - & & & & $\underline{1.10}^{\mathrm{c}}$ & & \\
\hline 51 & $\beta$-santalene & 1462 & 1460 & 1759 & & & $\underline{1.82}^{b}$ & & & \\
\hline 52 & allo-aromadendrene & 1463 & 1460 & - & & & & $\underline{0.70}^{\mathrm{c}}$ & & \\
\hline 53 & trans-cadina-1(6).4-diene & 1474 & 1477 & - & $0.10^{\mathrm{b}}$ & $0.25^{\mathrm{b}}$ & & & & \\
\hline 54 & $\gamma$-muurolene & 1477 & 1480 & 1691 & $0.17^{\mathrm{b}}$ & $0.28^{\mathrm{b}}$ & & & & \\
\hline 55 & sesquiterpene & 1480 & - & - & & & $\underline{1.08}^{\mathrm{c}}$ & & & \\
\hline 56 & germacrene D & 1485 & 1485 & 1714 & $2.66^{\mathrm{b}}$ & $16.05^{\mathrm{b}}$ & $2.87^{b}$ & $9.12^{\mathrm{b}}$ & $11.66^{\mathrm{b}}$ & $44.88^{\mathrm{b}}$ \\
\hline 57 & $\beta$-selinene & 1488 & 1490 & - & & & & $\underline{0.29^{\mathrm{c}}}$ & & \\
\hline 58 & trans-muurola-4(14),5-diene & 1493 & 1494 & 1729 & $\underline{0.15}^{\mathrm{b}}$ & & & & & \\
\hline 59 & bicyclogermacrene & 1500 & 1500 & 1739 & $9.73^{\mathrm{b}}$ & $22.93^{\mathrm{b}}$ & $5.44^{\mathrm{b}}$ & $7.63^{\mathrm{b}}$ & $9.33^{\mathrm{b}}$ & $30.44^{\mathrm{b}}$ \\
\hline 60 & $\alpha$-muurolene & 1506 & 1500 & 1798 & & & $0.20^{\mathrm{b}}$ & $0.10^{\mathrm{b}}$ & & \\
\hline 61 & $\beta$-bisabolene & 1508 & 1506 & 1775 & $0.43^{\mathrm{b}}$ & $4.45^{\mathrm{b}}$ & & & $0.58^{\mathrm{b}}$ & $1.71^{\mathrm{b}}$ \\
\hline 62 & sesquiterpene & 1510 & - & 1779 & & & $0.91^{\mathrm{c}}$ & $1.33^{\mathrm{c}}$ & & \\
\hline 63 & $\gamma$-cadinene & 1515 & 1514 & 1786 & $0.39^{\mathrm{b}}$ & $0.81^{\mathrm{b}}$ & $0.20^{\mathrm{b}}$ & $0.47^{\mathrm{b}}$ & & \\
\hline 64 & sesquiterpene & 1517 & - & - & & & & & $0.16^{\mathrm{c}}$ & $0.43^{\mathrm{c}}$ \\
\hline 65 & sesquiterpene & 1520 & - & - & $0.33^{\mathrm{c}}$ & $0.07^{\mathrm{c}}$ & & & & \\
\hline 66 & $\delta$-cadinene & 1524 & 1523 & 1762 & $2.06^{\mathrm{b}}$ & $2.79^{\mathrm{b}}$ & $0.91^{\mathrm{b}}$ & $1.29^{\mathrm{b}}$ & $0.38^{\mathrm{b}}$ & $1.03^{\mathrm{b}}$ \\
\hline 67 & $\begin{array}{l}\mathrm{nq}-145(100), 131(65), 187(59) \\
105(56), 202(45)\end{array}$ & 1532 & - & 1846 & 0.73 & 0.13 & & & & \\
\hline 68 & sesquiterpene & 1535 & - & - & & & $2.55^{\mathrm{c}}$ & $0.09^{\mathrm{c}}$ & & \\
\hline 69 & $\alpha$-cadinene & 1538 & 1539 & 1796 & $0.11^{\mathrm{b}}$ & $\operatorname{tr}^{\mathrm{b}}$ & & & & \\
\hline 70 & sesquiterpene & 1544 & - & 1791 & $0.12^{\mathrm{c}}$ & $0.80^{c}$ & & & & \\
\hline 71 & sesquiterpene & 1542 & - & 1779 & & & & & $0.09^{c}$ & $0.21^{\mathrm{c}}$ \\
\hline 72 & sesquiterpene & 1549 & - & 1879 & $0.36^{\mathrm{c}}$ & $0.30^{c}$ & & & & \\
\hline 73 & sesquiterpene & 1551 & - & 1880 & & & & & $0.18^{c}$ & $0.44^{\mathrm{c}}$ \\
\hline 74 & sesquiterpene & 1554 & - & 2015 & & & $0.83^{\mathrm{c}}$ & $0.20^{c}$ & & \\
\hline
\end{tabular}


Table 1. Continuation

\begin{tabular}{|c|c|c|c|c|c|c|c|c|c|c|}
\hline \multirow[b]{2}{*}{ No. } & \multirow[b]{2}{*}{ Compound } & \multirow{2}{*}{$\begin{array}{l}\text { LTPRI } \\
\text { OV5 }\end{array}$} & \multirow{2}{*}{$\begin{array}{l}\text { LTPRI } \\
\text { Lit. }^{20}\end{array}$} & \multirow[b]{2}{*}{ LTPRI Wax } & \multicolumn{2}{|c|}{ B. punctulata } & \multicolumn{2}{|c|}{ B. dracunculifolia } & \multicolumn{2}{|c|}{ E. laevigatum } \\
\hline & & & & & $\begin{array}{l}\text { HD } \\
\% \text { area }\end{array}$ & $\begin{array}{l}\text { SPME } \\
\text { \%area }\end{array}$ & $\begin{array}{l}\text { HD } \\
\% \text { area }\end{array}$ & $\begin{array}{l}\text { SPME } \\
\text { \%area }\end{array}$ & $\begin{array}{c}\text { HD } \\
\% \text { area }\end{array}$ & $\begin{array}{l}\text { SPME } \\
\text { \%area }\end{array}$ \\
\hline $\begin{array}{l}\text { sesqu } \\
\text { sesqu }\end{array}$ & $\begin{array}{l}\text { iterpenes and oxygenated } \\
\text { iterpenes }\end{array}$ & & & & 54.76 & 20.21 & 45.87 & 22.85 & 71.79 & 16.57 \\
\hline 75 & $(E)$-nerolidol & 1562 & 1563 & 1028 & $0.28^{\mathrm{b}}$ & $0.11^{\mathrm{b}}$ & $22.16^{\mathrm{b}}$ & $12.80^{\mathrm{b}}$ & & \\
\hline 76 & oxygenated sesquiterpene & 1569 & - & 2084 & $0.32^{\mathrm{c}}$ & $0.07^{\mathrm{c}}$ & $1.07^{\mathrm{c}}$ & $0.76^{c}$ & & \\
\hline 77 & oxygenated sesquiterpene & 1579 & - & 2068 & $0.87^{\mathrm{c}}$ & $0.86^{\mathrm{c}}$ & & & & \\
\hline 78 & spathulenol & 1580 & 1578 & 2099 & $0.88^{\mathrm{b}}$ & $0.24^{\mathrm{b}}$ & $8.81^{\mathrm{b}}$ & $3.31^{\mathrm{b}}$ & $0.30^{\mathrm{b}}$ & $0.42^{\mathrm{b}}$ \\
\hline 79 & globulol & 1585 & 1585 & 2094 & $1.05^{\mathrm{b}}$ & $0.23^{\mathrm{b}}$ & & & & \\
\hline 80 & sesquiterpene & 1589 & - & 2092 & & & & & $0.26^{c}$ & $0.12^{\mathrm{c}}$ \\
\hline 81 & $\beta$-copaen-4- $\alpha$-ol & 1589 & 1591 & 2159 & & & $3.39^{\mathrm{b}}$ & $2.10^{\mathrm{b}}$ & & \\
\hline 82 & guaiol & 1594 & 1601 & 2102 & $3.43^{\mathrm{b}}$ & $2.06^{\mathrm{b}}$ & & & & \\
\hline 83 & viridiflorol & 1597 & 1593 & 2162 & & & $3.54^{\mathrm{b}}$ & $1.12^{\mathrm{b}}$ & & \\
\hline 84 & oxygenated sesquiterpene & 1600 & - & & $0.28^{\mathrm{c}}$ & $0.10^{\mathrm{c}}$ & & & & \\
\hline 85 & oxygenated sesquiterpene & 1605 & - & 2128 & $\underline{\mathbf{0 . 3 2}}^{\mathrm{c}}$ & & & & & \\
\hline 86 & oxygenated sesquiterpene & 1608 & - & 2198 & & & $1.71^{\mathrm{c}}$ & $0.87^{\mathrm{c}}$ & & \\
\hline 87 & oxygenated sesquiterpene & 1620 & - & 2136 & $0.24^{\mathrm{c}}$ & $0.06^{\mathrm{c}}$ & & & & \\
\hline 88 & $\begin{array}{l}\mathrm{nq}-119(100), 161(66), 159(57) \\
105(54), 121(22)\end{array}$ & 1624 & - & 2219 & 1.56 & 0.57 & & & & \\
\hline 89 & oxygenated sesquiterpene & 1631 & - & 2187 & & & $0.56^{\mathrm{c}}$ & $0.34^{c}$ & & \\
\hline 90 & cis-cadin-4-en-7-ol & 1636 & 1637 & 2116 & $6.77^{\mathrm{b}}$ & $2.74^{\mathrm{b}}$ & & & $0.31^{\mathrm{b}}$ & $0.18^{\mathrm{b}}$ \\
\hline 91 & oxygenated sesquiterpene & 1643 & - & - & & & $0.63^{c}$ & $0.40^{c}$ & & \\
\hline 92 & oxygenated sesquiterpene & 1643 & - & 2185 & $1.99^{\mathrm{c}}$ & $0.13^{\mathrm{c}}$ & & & & \\
\hline 93 & oxygenated sesquiterpene & 1645 & - & 2203 & & & $1.01^{\mathrm{c}}$ & & & \\
\hline 94 & torreyol & 1645 & 1646 & 2147 & $0.53^{\mathrm{b}}$ & $0.10^{\mathrm{b}}$ & & & $0.47^{\mathrm{b}}$ & $0.14^{\mathrm{b}}$ \\
\hline 95 & $\alpha$-cadinol & 1656 & 1654 & 2211 & $3.17^{\mathrm{b}}$ & $0.10^{\mathrm{b}}$ & & & & \\
\hline 96 & oxygenated sesquiterpene & 1658 & - & 2217 & & & $1.45^{\mathrm{c}}$ & $0.49^{c}$ & & \\
\hline 97 & oxygenated sesquiterpene & 1659 & - & 2149 & & & & & $0.61^{\mathrm{c}}$ & $0.15^{\mathrm{c}}$ \\
\hline 98 & sesquiterpene $(201,216)$ & 1670 & - & 2166 & $14.67^{\mathrm{c}}$ & $8.36^{\mathrm{c}}$ & & & $2.31^{\mathrm{c}}$ & $0.91^{\mathrm{c}}$ \\
\hline 99 & (epi- $\alpha$ ) bisabolol & 1685 & 1685 & 2250 & $3.08^{\mathrm{b}}$ & $0.70^{\mathrm{b}}$ & & & & \\
\hline 100 & (Z)-farnesol & 1686 & 1686 & 2234 & & & & & $0.45^{\mathrm{b}}$ & $0.16^{\mathrm{b}}$ \\
\hline 101 & oxygenated sesquiterpene & 1693 & - & 2391 & $2.20^{\mathrm{c}}$ & $2.41^{\mathrm{c}}$ & $0.65^{\mathrm{c}}$ & $0.11^{\mathrm{c}}$ & & \\
\hline 102 & oxygenated sesquiterpene & 1699 & - & 2255 & $2.83^{\mathrm{c}}$ & $0.96^{\mathrm{c}}$ & & & & \\
\hline 103 & sesquiterpene & 1700 & - & 2242 & & & & & $0.64^{c}$ & $0.12^{\mathrm{c}}$ \\
\hline 104 & oxygenated sesquiterpene & 1707 & - & & $0.75^{\mathrm{c}}$ & $0.19^{\mathrm{c}}$ & & & & \\
\hline 105 & oxygenated sesquiterpene & 1718 & - & & & & $0.23^{\mathrm{c}}$ & $0.32^{\mathrm{c}}$ & & \\
\hline 106 & oxygenated sesquiterpene & 1722 & - & 2298 & ${\underline{0.82^{c}}}^{\mathrm{c}}$ & & & & & \\
\hline 107 & $\begin{array}{l}\mathrm{nq}-143(100), 185(92), 129(64) \\
128(55), 157(54)\end{array}$ & 1727 & - & & 0.71 & 0.11 & & & & \\
\hline 108 & sesquiterpene $(212,197)$ & 1735 & - & & 1.09 & 0.22 & & & & \\
\hline 109 & sesquiterpene $(214,199)$ & 1738 & - & 2333 & $\underline{0.75}$ & & & & & \\
\hline 110 & laevigatin & 1738 & - & $2404^{e}$ & & & & & $59.63^{\mathrm{c}}$ & $13.82^{\mathrm{c}}$ \\
\hline 111 & oxygenated sesquiterpene & 1739 & - & - & & & $0.38^{\mathrm{c}}$ & $0.13^{\mathrm{c}}$ & & \\
\hline 112 & sesquiterpene $(214,199)$ & 1747 & - & - & & & $0.28^{\mathrm{c}}$ & $0.09^{c}$ & & \\
\hline 113 & sesquiterpene & 1751 & - & 2357 & $7.14^{\mathrm{c}}$ & $0.38^{\mathrm{c}}$ & & & & \\
\hline
\end{tabular}


Table 1. Continuation

\begin{tabular}{|c|c|c|c|c|c|c|c|c|c|c|}
\hline \multirow[b]{2}{*}{ No. } & \multirow[b]{2}{*}{ Compound } & \multirow{2}{*}{$\begin{array}{l}\text { LTPRI } \\
\text { OV5 }\end{array}$} & \multirow{2}{*}{$\begin{array}{l}\text { LTPRI } \\
\text { Lit. }^{20}\end{array}$} & \multirow[b]{2}{*}{ LTPRI Wax } & \multicolumn{2}{|c|}{ B. punctulata } & \multicolumn{2}{|c|}{ B. dracunculifolia } & \multicolumn{2}{|c|}{ E. laevigatum } \\
\hline & & & & & $\begin{array}{c}\text { HD } \\
\% \text { area }\end{array}$ & $\begin{array}{l}\text { SPME } \\
\% \text { area }\end{array}$ & $\begin{array}{l}\text { HD } \\
\% \text { area }\end{array}$ & $\begin{array}{l}\text { SPME } \\
\% \text { area }\end{array}$ & $\begin{array}{l}\mathrm{HD} \\
\% \text { area }\end{array}$ & $\begin{array}{l}\text { SPME } \\
\% \text { area }\end{array}$ \\
\hline 114 & sesquiterpene $(214,199)$ & 1753 & - & 2252 & & & & & $3.11^{\mathrm{c}}$ & $0.42^{\mathrm{c}}$ \\
\hline 115 & sesquiterpene $(214,199)$ & 1775 & - & 2359 & $2.86^{\mathrm{c}}$ & $0.33^{\mathrm{c}}$ & & & $1.07^{\mathrm{c}}$ & $0.08^{\mathrm{c}}$ \\
\hline 116 & sesquiterpene & 1785 & - & - & $0.19^{\mathrm{c}}$ & $0.06^{\mathrm{c}}$ & & & & \\
\hline 117 & $\begin{array}{l}\mathrm{nq}-68(100), 57(89), 82(78), \\
95(69), 69(64)\end{array}$ & 1837 & - & - & 0.06 & $\operatorname{tr}$ & & & & \\
\hline 118 & sesquiterpene $(212,197)$ & 1891 & - & - & $0.10^{\mathrm{c}}$ & $\operatorname{tr}^{\mathrm{c}}$ & & & & \\
\hline 119 & $\begin{array}{l}\mathrm{nq}-210(100), 209(35), 195(28) \\
165(27), 167(16)\end{array}$ & 1917 & - & - & 0.12 & $\operatorname{tr}$ & & & & \\
\hline 120 & $\begin{array}{l}\text { nq - 217(100), 189(35), 164(28), } \\
\text { 157(27), 95(16) }\end{array}$ & 1924 & & & & & & & 0.33 & 0.11 \\
\hline 121 & sesquiterpene $(197,212)$ & 2069 & - & - & & & & & $2.64^{c}$ & $\operatorname{tr}^{\mathrm{c}}$ \\
\hline 122 & $\begin{array}{l}\text { nq }-82(100), 57(98), 71(93), \\
95(92), 68(92)\end{array}$ & 2140 & - & - & & & & & 1.05 & \\
\hline
\end{tabular}

nq: not quantified; empty cell means the compound was not detected or it was not possible to get its LTPRI due to co-elutions, similarity of mass spectra among several isomers, or lack of such information in the scientific literature; ( ):some of the major ions in the mass spectrum of nq compounds; -: whenever it was not possible to determine the LTPRI experimentally, as they were determined mainly for hydrodistilled oils; tr: compounds detected as traces level; compounds name written in bold and italics were detected either in the hydrodistilled oil or using SPME. Area \% of these compounds is

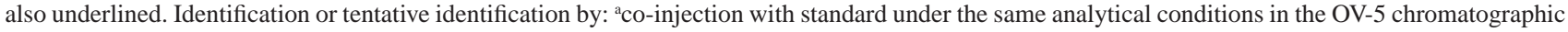
column; ${ }^{b}$ comparison of experimantal LTPRI with the ones found in the literature; ${ }^{20}$ comparison of experimental retention and mass spectra data with literature data; ${ }^{20 \mathrm{~d}}$ Pino et al. ${ }^{41}$; ${ }^{\mathrm{e}}$ Maia et al.,${ }^{19}$.

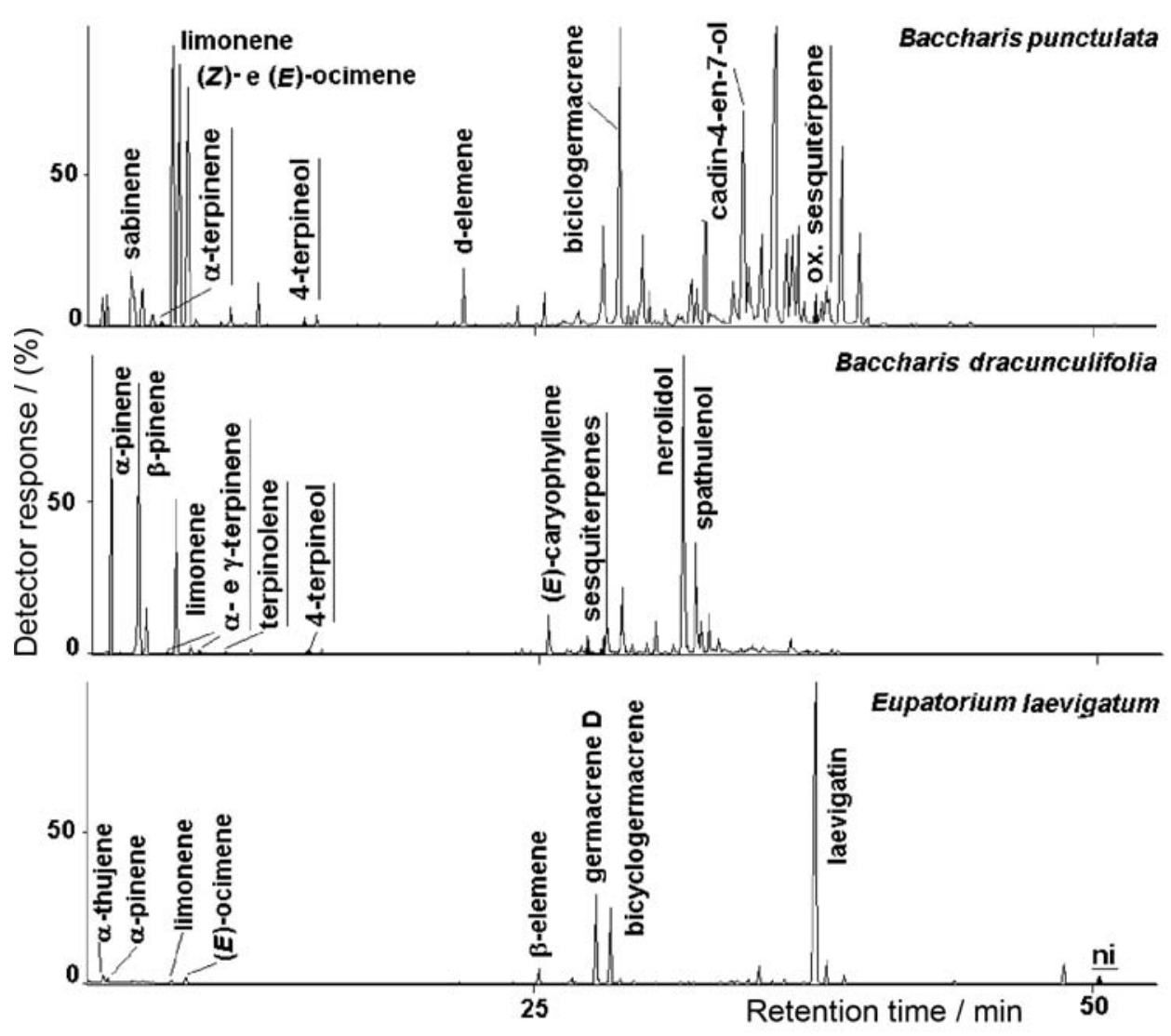

Figure 1. Chromatographic profile of the essential oils of Baccharis punctulata, Baccharis dracunculifolia, and Eupatorium laevigatum. 


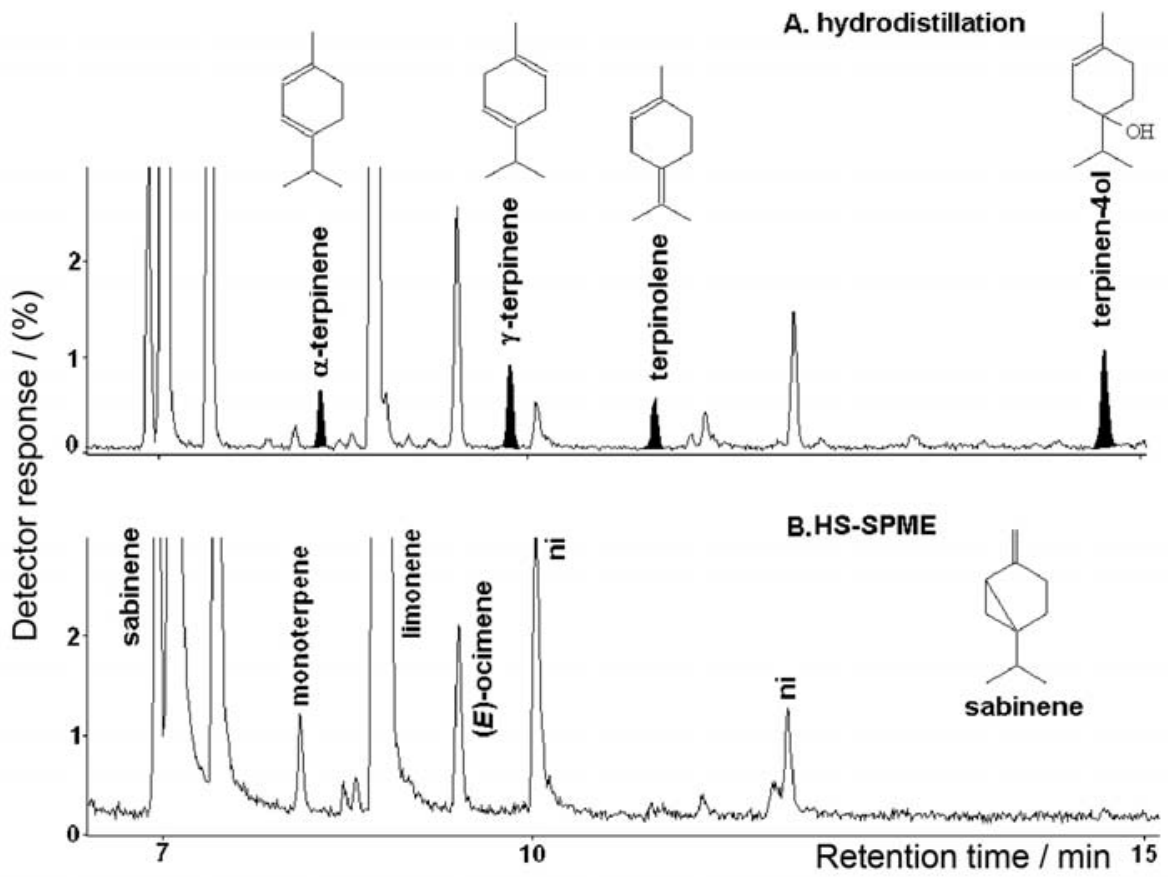

Figure 2. Part of the chromatographic profile of B. dracunculifolia hydrodistilled oil (A) and of the HS-SPME (B) of the chopped leaves of the same plant, showing marked peaks detected either by hydrodistillation or HS-SPME.
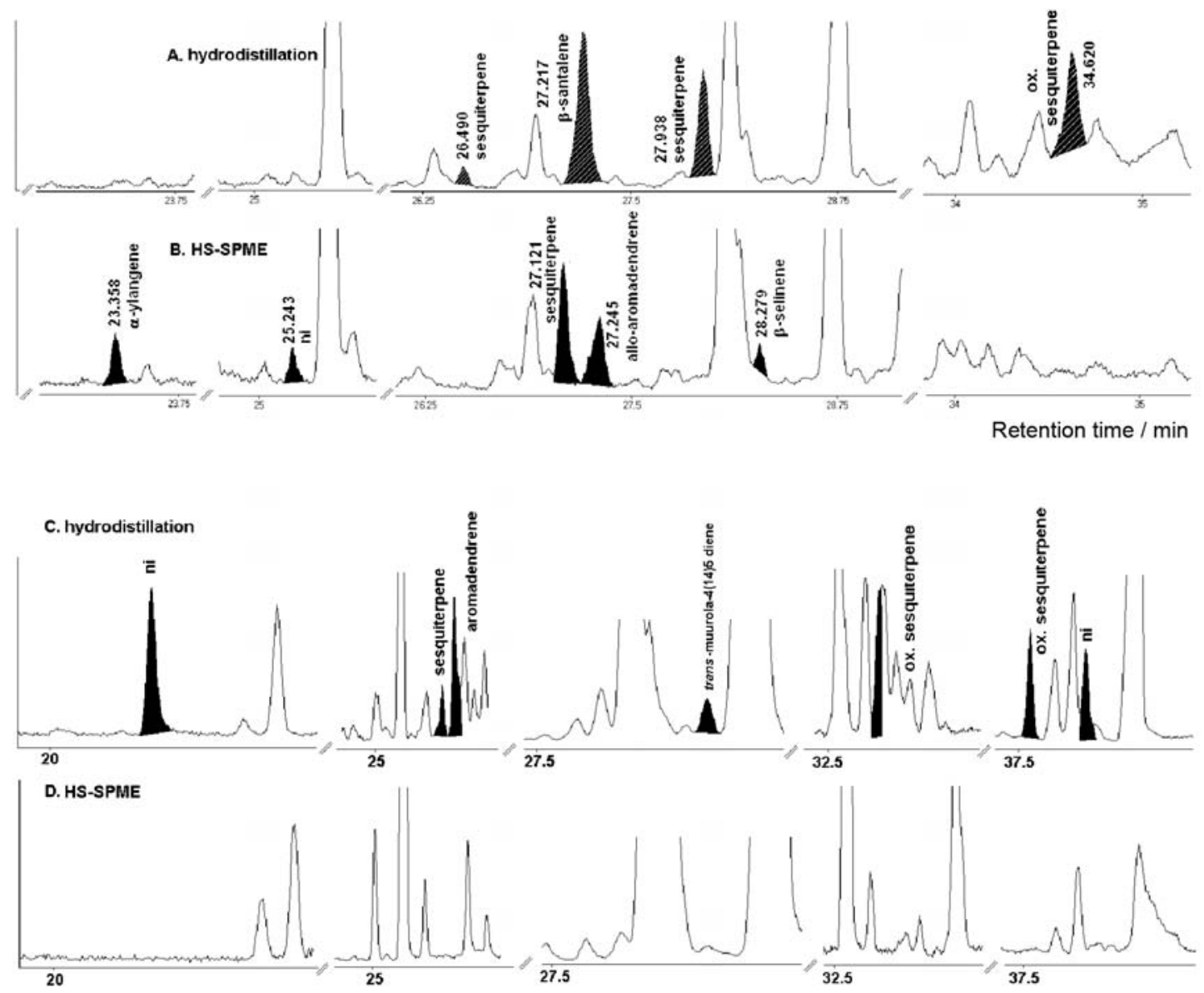

Figure 3. Part of the chromatographic profile of the hydrodistilled oils of B. dracunculifolia (A) and of B. punctulata (C) and of the HS-SPME of the chopped leaves of the same plants of B. dracunculifolia (B) and of B. punctulata (D), showing the marked peaks detected only by one of the techniques. 
A reasonable explanation for the presence or absence of some of these compounds may not be straightforward, as sesquiterpene hydrocarbons undergo several rearrangements, which can be thermal, photochemical or acid-catalyzed. Chemical transformations of sesquiterpene compounds are complex processes, which may include oxidation, thermal decomposition or hydrolysis, being affected by many parameters, such as light, $\mathrm{pH}$, and temperature. ${ }^{35}$ Only HD detected some oxygenated compounds, and their presence may be understood as products of thermal oxidation occurring during the distillation process. ${ }^{34}$ This type of transformation has already been observed in some other plant species containing thermally sensitive compounds, when conventional extraction processes using higher temperatures were employed. ${ }^{4,16,21}$

On the other side, some compounds were found only in the headspace of chopped leaves and were not detected in the hydrodistilled oil as is shown in Figure 4B (10 peaks: 5 sequiterpenes, 3 hexenyl esters, coumaran, and coumarin) where chromatographic peaks were marked in dark ink.

Figure $4 \mathrm{~A}$ shows the corresponding chromatogram of the essential oil of the same plant. The presence of the so called green leaf volatiles (in this case hexenyl esters) are due to enzymatic cleavage of non volatile precursors, which was prompt by mechanical damage of fresh leaves, and has already been observed in former works when SPME was employed as extraction technique. ${ }^{1,4,34}$ The presence of coumarin (1,2-benzopyrone or 2H-1-benzopyran-2-one) and coumaran (2,3-dihydrobenzofuran) is for the first time reported among volatile components of E. laevigatum leaves. ${ }^{19}$ Unlike other simple coumarins, the biosynthesis of the coumarin itself is obscure. As far as it is known it is a derivative of trans-cinnamic acid (shikimate pathway), although it remains uncertain if 2-H-1-benzopyran-2one is a true plant metabolite of the shikimic pathway or if it rises from chemical transformation of another precursor. This last hypothesis is considered plausible for Melilotus sp. (Fabaceae), since in this case the glucoside of 2'-hydroxycinnamic acid (coumarinic acid) rather than the coumarin is the true plant metabolite. ${ }^{36}$

The presence of coumarin in the headspace of $E$. laevigatum leaves is consistent with the chemotaxonomic profile of the Asteraceae family. First, species that belong to evolved families as Asteraceae have a general tendency to lack the shikimate derivatives in detriment of mevalonate derivatives and this tendency is also observed in the volatile chemistry. ${ }^{37}$ Secondly, although the presence of 2-H-1-benzopyran-2-one is not common in other Asteraceae species, which mainly produce oxygenated coumarins, this plant group is one of the major coumarin producers among angiosperms and is highly specialized in the biosynthesis of simple coumarins. ${ }^{38}$

Coumarin may be a signaling compound among other plant species or even arthropods species. It is reported that this arylpropanoid completely inhibited the growth of alfafa (Medicago sativa) and bayard grass (Echinochloa crus-galli, var. oryzicola), what means it may be used for natural weed control. ${ }^{39}$ Coumarin is also an attractant to herbivore insects, such as Listroderes costirostris (Coleoptera: Curculionidae) and Sitona cylindricollis (Coleoptera:Curculionidae). ${ }^{40}$ On the other hand, coumarin is one of the feed deterrents produced by Trifolium glanduliferum (Fabaceae) and is probably responsible for the resistance of this plant against the red-legged earth mite Halotydeus destructor (Acari: Penthaleidae).

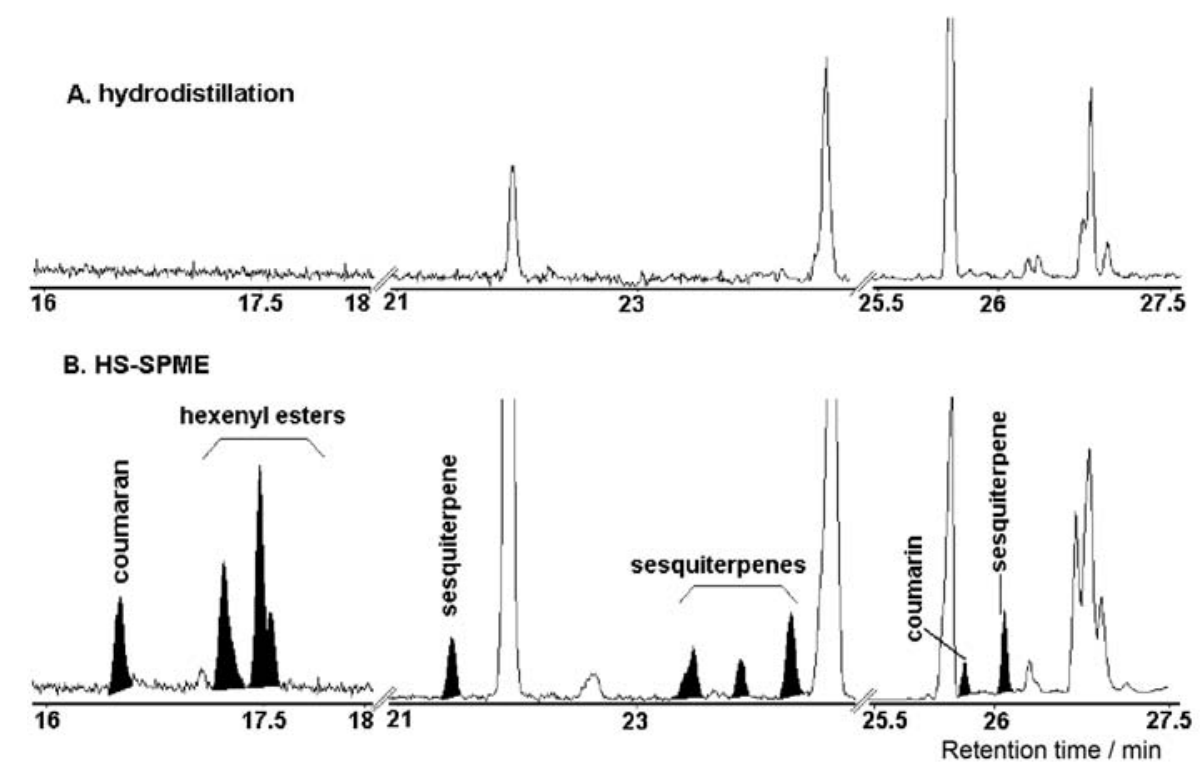

Figure 4. Chromatogram of volatile components of young leaves of E. laevigatum. 


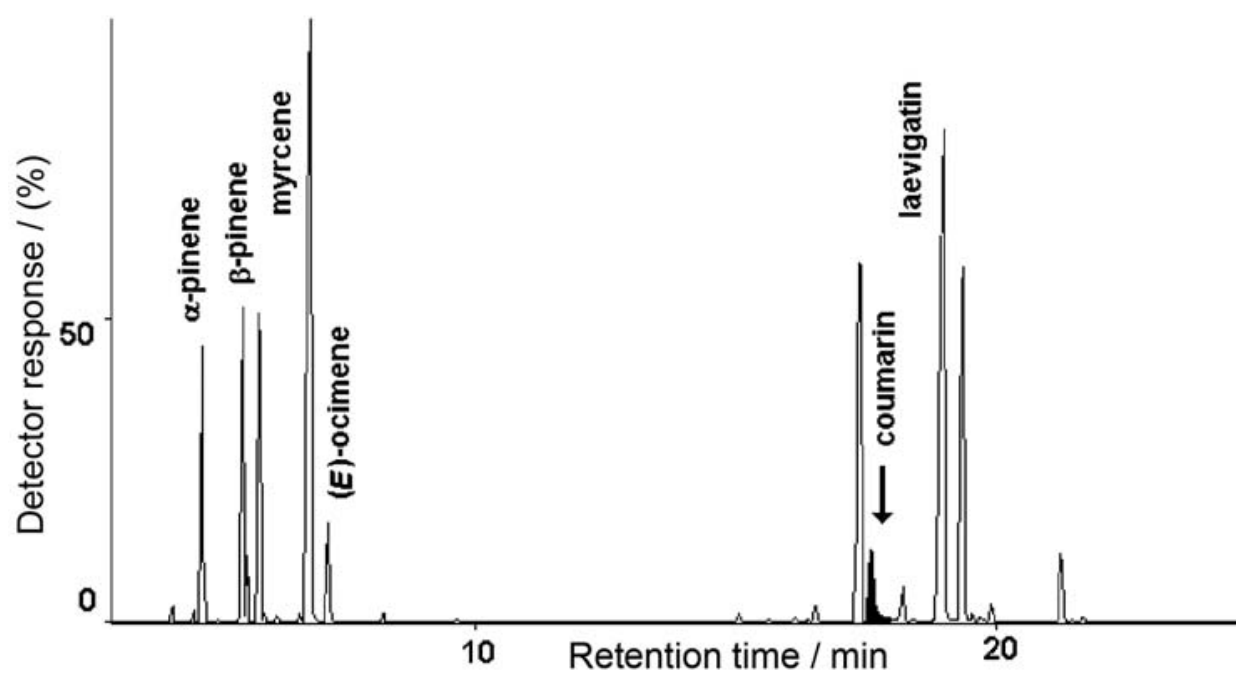

Figure 5. Chromatogram of volatile components of adult leaves of another individual of E. laevigatum.

Figure 5 presents a chromatogram of volatile components of adult leaves of another sample of E. laevigatum.

This additional analysis confirms the presence of coumarin in adult leaves of E. laevigatum and reinforces the results of experiments with young leaves, which were performed as five replicates.

The compound tentatively identified as coumaran was detected in the headspace of young leaves of E. laevigatum (Figure 4B), but was not in the headspace of adult leaves of the E. laevigatum (Figure 5). This fact can be explained by genetic, ontogenetic or ecological differences between samples and should be further investigated in order to elucidate the role of this substance as a signal. Its LTPRI on a SPB5 chromatographic column is reported to be $1224 .{ }^{41}$ In this work it was not possible to experimentally determine the LTPRI of this compound, as the indices were only calculated for the essential oil components. However, coumaran eluted in the proper chromatographic region (between LTPRI 1189 ( $\alpha$-terminal) and 1251 (trans-geraniol)), and presented a $90 \%$ match with the mass spectrum of the $6^{\text {th }}$ edition of Wiley mass spectra library. This compound may also play an ecological role as feed deterrant as it was found in Cyperus nipponicus (Cyperaceae) as responsible for the inhibition of polyphagous insects (Spodoptera litura (Lepidoptera: Noctuidae) feeding. ${ }^{42}$

\section{Conclusions}

A complete characterization of volatile components of plants may require the use of more than one extraction technique, as different principles of extraction and distinct extraction parameters (temperature, $\mathrm{pH}$, solvent, etc) may contribute to various chromatographic profiles. Qualitative differences between hydrodistilled essential oils and the volatile compounds found in the headspace of B. punctulata, $B$. dracunculifolia, and E. laevigatum chopped leaves brought additional information about their composition and their possible chemical transformation during hydrodistillation process. The fact that some compounds were detected only by HS-SPME might possibly unveil their infochemical roles regarding plant defense of some species.

\section{Acknowledgments}

We thank Supelco for the SPME fibers supply. Students' scholarships from PROPESQ-UFRGS, FAPERGS, $\mathrm{CNPq}$ are acknowledged. We thank Prof. Dr. Nelson Ivo Matzenbacher for plant sampling and identification and also Andreia L. Silva for the first sampling of E. laevigatum.

\section{References}

1. Augusto, F.; Lopes, A. L. e.; Zini, C. A.; TrAC, Trends Anal. Chem. 2003, 22, 160.

2. Dudavera, N.; Pichersky, E.; Plant Physiol. 2000, 122, 627.

3. Stashenko, E. E.; Jaramilo, B. E.; Martinéz, J. R.; J. Chromatogr., A 2004, 1025, 93.

4. Zini, C. A.; Zanin, K. D.; Christensen, E.; Caramão, E. B.; Pawliszyn, J.; J. Agric. Food Chem. 2003, 51, 2679.

5. Zini, C. A.; Augusto, F.; Christensen, E.; Caramão, E. B.; Pawliszyn, J.; J. Agric. Food Chem. 2002, 50, 7199.

6. Flamini, G.; Cioni, P. L.; Morelli, I.; J. Agric. Food Chem. 2002, 50, 4647.

7. Flamini, G.; Cioni, P. L.; Morelli, I.; Food Chem. 2005, 91, 63.

8. Jirovetz, L.; Smith, D.; Buchbauer, G.; J. Agric. Food Chem. 2002, 50, 4643. 
9. Bertoli, A.; Menichini, F.; Mazzetti, M.; Spinelli, G.; Morelli, I.; Flavour Fragr. J. 2003, 18, 91.

10. Flamini, G.; Cioni, P. L.; Morelli, I.; Maccioni, S.; Baldini, R.; Food Chem. 2004, 85, 599.

11. Lamikanra, O.; Richard, O. A.; J. Sci. Food Agric. 2004, 84, 1812.

12. Bertoli, A.; Menichini, F.; Noccioli, C.; Morelli, I.; Pistelli, L.; Flavour Fragr. J. 2004, 19, 166.

13. Palá-Paúl, J.; Brophy, J. J.; Goldsack, R. J.; Fontaniella, B.; Biochem. Syst. Ecol. 2004, 32, 55.

14. Bertolli, A.; Pistelli, L.; Morelli, I.; Fraternale, D.; Giampieri, L.; Ricci, D.; Flavour Fragr. J. 2004, 19, 522.

15. Choi, H. S.; J. Agric. Food Chem. 2004, 52, 8099.

16. Wongpornchai, S.; Sriseadka, T.; Choonvisase, S.; J. Agric. Food Chem. 2003, 51, 457.

17. Cassel, E.; Frizzo, C. D.; Vanderlinde, R.; Atti-Serafini, L.; Lorenzo, D.; Dellacassa, E.; Ind. Eng. Chem. Res. 2000, 39, 4803 .

18. Loayza, I.; Abudjer, D.; Aranda, R.; Jakupovic, J.; Collin, G.; Deslauriers, H.; Jean, F. I.; Phytochemistry 1995, 38, 381.

19. Maia, J. S.; Zoghbi, M. B.; Andrade, E. H. A.; Silva, M. H. L.; Luz, A. I. R.; Silva, J.; Biochem. Syst. Ecol. 2002, 30, 1071.

20. Adams, R. P.; Identification of Essential Oil Components by Gas Chromatography/Quadrupole Mass Spectrometry; Allured Publishing Corporation: Illinois, 2001.

21. Liang, M.; Qi, M.; Zhang, C.; Zhou, S.; Fu, R.; Huang, J.; Anal. Chim. Acta 2005, 531, 97.

22. Rohloff, J.; Phytochemistry 2002, 59,655.

23. Buttery, R. G.; Ling, L. C.; J. Agric. Food Chem. 1985, 33, 772.

24. Liu, S. H.; Norris, D. M.; Lyne, P.; J. Agric. Food Chem. 1989, $37,496$.

25. Anderson, P.; Hansson, B. S.; Löfqvist, J.; Physiol. Entomol. 1995, 20, 189.
26. Blight, M. M.; Pickett, J. A.; Wadhams, L. J.; Woodcock, C. M.; J. Chem. Ecol. 1995, 21, 1649.

27. Dickens, J. C.; Chem. Senses 1990, 15, 311.

28. Hansson, B. S.; Larsson, M. C.; Leal, W. S.; Physiol. Entomol. 1999, 24, 121.

29. Jönsson, M.; Anderson, P.; Physiol. Entomol. 1999, 24, 377.

30. Todd, J. L.; Baker, T. C.; Naturwissenschaften 1993, 80, 183.

31. Wibe, A.; Borg-Karlson, A. K.; Norin, T.; Mustaparta, H.; J. Comp. Physiol. 1997, 180, 585.

32. Schütz, S.; Weissbecker, B.; Hummel, H. E.; Apel, K. H.; Bleckmann, H.; Nature 1999, 398, 287.

33. Cooper, M. A.; Holden, C. M.; Loftus, P. ; Whittaker, D.; J. Chem. Soc., Perkin II 1973, 665.

34. Koedam, A. In Capillary Gas Chromatography in Essential Oil Analysis; Sandra, P., Bicchi, C., eds.; Huethig Verlag: New York, 1987.

35. Garnero, J.; Tabacchi, R. In Capillary Gas Chromatography in Essential Oil Analysis; Sandra, P., Bicchi, C., eds.; Huethig Verlag: New York, 1987.

36. Wink, M.; Biochemistry of Plant Secondary Metabolism; Sheffield Academic Press: Sheffield, 1999.

37. Gottlieb, O. R.; Salatino, A.; Cienc. Cult. 1987, 39, 707.

38. Ribeiro, C. V. C.; Kaplan, M. A. C.; Quim. Nova 2002, 25 , 533.

39. Chon, S. U.; Kim, Y. M.; J. Agron. Crop. Sci. 2004, 190, 145.

40. Metcalf, R. L.; Metcalf, E. R.; Plant Kairomones in Insect Ecology and Control; Chapman and Hall: London, 1992.

41. Pino, J.; Marbot, R.; Rosado, A.; Flavour Fragr. J. 2002, 17, 401.

42. Morimoto, M.; Urakawa, M.; Fujitaka, T.; Komai, K.; Biosci., Biotechnol., Biochem. 1999, 63, 840.

Received: March 7, 2008

Web Release Date: December 18, 2008 